\title{
Mais quand pourrai-je enfin jeter ce vieux dossier médical?
}

\section{Valérie Junod}

Professeure à la Faculté de droit de I'UNIGE et à la Faculté des HEC de I'UNIL

Combien de temps un médecin doit-il garder le dossier médical de ses patients et patientes? La loi fédérale n'imposant aucune durée de conversation minimale ou maximale, la question reste largement ouverte. Ce flou est renforcé par la récente révision du délai de prescription, passant de dix à vingt ans, et l'introduction du dossier électronique du patient.

Les professionnels de la santé sont tenus de maintenir à jour un dossier médical pour chaque patient. Ce dossier permet le suivi longitudinal du patient en cas de soins au long cours. Il facilite la communication avec d'autres professionnels de la santé avec, à la clé, une meilleure coordination et efficacité des soins. Sous l'angle juridique, un dossier médical bien tenu aide le médecin à démontrer qu'il a agi conformément aux règles de l'art médical et de la science. Il lui permet aussi de prouver qu'il a dûment informé son patient avant chaque intervention, ou en tout cas avant chaque intervention significative, de sorte que le patient a pu donner son consentement éclairé.

\section{A la fois précieux et fastidieux}

Entrer les données nécessaires dans un dossier médical peut être une tâche administrative fastidieuse. Et plus le dossier est complet, plus il est précieux, mais plus il faut y consacrer du temps [1]. De surcroît, avec l'essor escompté du dossier électronique du patient (DEP), le dossier médical, ou du moins une partie de celui-ci, jouira d'une plus grande diffusion. Le patient pourra le consulter et «l'ouvrir» à qui il entend, sans que son médecin n'ait son mot à dire.

La période pendant laquelle le dossier médical doit être conservé reste une question encore largement ouverte [2].

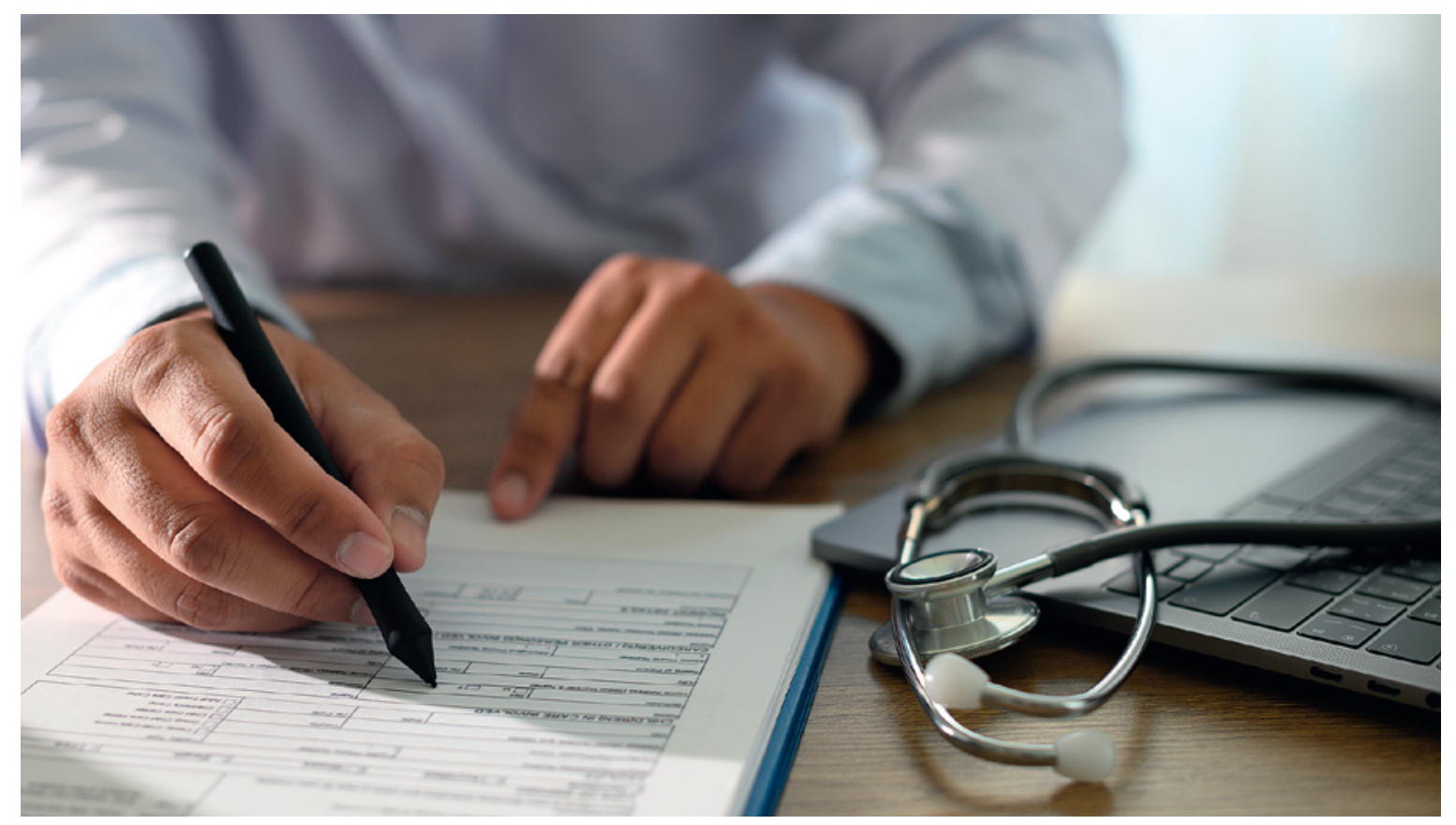

Plus un dossier médical est complet, plus il est précieux (image symbolique, (c) One Photo | Dreamstime.com). 
Tant que le patient est suivi par le même médecin, il peut s'attendre à ce que celui-ci ait son dossier médical complet. On pense notamment au pédiatre qui a suivi un enfant de sa naissance à sa majorité; détruire les données concernant les cinq premières années de vie au moment où l'adolescent atteint ses 15 ans serait contraire aux intérêts du patient. En effet, les maladies soignées, les vaccins administrés, les séquelles sont des informations précieuses pour l'adolescent et le futur adulte. Il en va de même pour les soins gériatriques: lorsque la mémoire du patient peut, avec l'âge, flancher, un dossier gardé sur le long terme est hautement utile. Les patients ayant subi des maladies sérieuses sujettes à rechute ont eux aussi besoin d'un dossier complet.

\section{Quelle durée de conservation?}

Juridiquement, la situation est complexe. De multiples lois et ordonnances fédérales et cantonales, de droit public ou privé, ont vocation à s'appliquer. La législation fédérale n'impose pas une durée de conservation minimale ou maximale des dossiers médicaux. Généralement, la durée minimale est déduite des délais de prescription applicables aux actions en responsabilité civiles (contractuelle ou délictuelle) et pénales des patients contre leurs soignants. La raison est que le soignant doit pouvoir se défendre en s'appuyant sur le dossier médical; inversement, le client peut demander une reddition de comptes, à savoir un compte rendu détaillé des soins administrés ou omis. Les patients ont en outre un droit d'accès à leurs données personnelles et peuvent donc utiliser le dossier médical pour fonder une action en justice. Avant 2020, le délai absolu de prescription civile était de dix ans à compter de l'acte dommageable (soin mal

\section{L'essentiel en bref}

- Les médecins doivent tenir à jour un dossier médical pour chaque patient. En plus du suivi et de la coordination des soins, il permet de prouver que le soignant a bien agi et donc de se défendre en cas de soin mal effectué.

- La durée de conservation - minimale et maximale - du dossier n'est pas fixée par la loi au niveau fédéral. Généralement, la durée minimale est déduite des délais de prescription applicables aux actions civiles et pénales.

- En 2020, le délai absolu de prescription civile est passé de dix à vingt ans à partir de l'acte dommageable. Les médecins ont ainsi intérêt à garder un dossier pendant au moins vingt ans.

- Le droit cantonal peut prescrire des délais de conservation minimale, voire maximale. Si ces délais sont plus courts, ils ne l'emportent pas sur le droit fédéral.

- Le dossier électronique du patient (DEP) peut aussi influer sur la durée de conservation. Les données du patient étant partagées plus largement, il est délicat pour le médecin de détruire définitivement un dossier même après vingt ans. effectué, intervention faite sans consentement, action indument omise). Ce délai pouvait être interrompu autant de fois que nécessaire par le patient lésé, entrâ̂nant l'octroi d'un nouveau délai complet.

\section{Vingt ans de conservation préconisée}

Début 2020, le délai de prescription s'est vu prolonger à la suite de la révision du Code des obligatons (CO). Passant de dix à vingt ans, il s'applique lorsque le demandeur se plaint d'une atteinte à son intégrité corporelle ou lorsqu'il est décédé en raison de l'acte ou de l'omission du défendeur. Ce délai prolongé ne s'applique donc pas à toutes les actions en responsabilité des patients contre leurs médecins [3]. En sus, le patient - ou ses proches en cas de décès - doit désormais respecter un délai relatif de trois ans dès qu'il connaît qui a causé quel dommage.

Avec la révision du CO, tous les professionnels de la santé ont aujourd'hui intérêt à garder les dossiers pendant au moins vingt ans à compter de la dernière mesure ayant pu faire naître une responsabilité. Parfois même plus longtemps, si l'examen du bien-fondé de cette mesure litigieuse demande l'analyse d'informations (encore) plus anciennes du dossier. N'incluant aucune solution transitoire, le changement de régime déploie ses effets même pour des dossiers ouverts sous l'ancien droit.

Ces différents délais découlent, on l'a vu, du Code des obligations. Toutefois, le droit pénal peut également jouer un rôle. Lorsque le délai de prescription de droit pénal est plus long que celui de droit civil, c'est le premier qui s'applique aux deux actions, pénale et civile [4]. En cas de reproches d'homicide ou de lésions corporelles par négligence, le délai pénal est de dix ans. Si ce délai est inférieur aux vingt ans du CO, il permet au patient de

\section{Das Wichtigste in Kürze}

- Das Patientendossier dient neben der Überwachung und Koordination der Behandlung dem Nachweis, dass die Pflegeperson korrekt gehandelt hat, und somit der Selbstverteidigung in dem Falle, dass die Behandlung nicht die gewünschten Ergebnisse zeigt.

- Die minimale und maximale Aufbewahrungsdauer des Dossiers ist auf Bundesebene nicht gesetzlich festgelegt. In der Regel wird die Mindestdauer von den für zivil- und strafrechtliche Verfahren geltenden Verjährungsfristen abgezogen.

- 2020 wurde die absolute zivilrechtliche Verjährungsfrist von zehn auf zwanzig Jahre ab dem Datum der schädigenden Handlung verlängert. Es liegt daher im Interesse der Ärzte, Unterlagen mindestens zwanzig Jahre lang zu bewahren.

- Das kantonale Recht kann Aufbewahrungsfristen vorschreiben. Das Bundesrecht gilt, wenn die kantonalen Fristen kürzer sind.

- Auch das elektronische Patientendossier (EPD) kann die Aufbewahrungsdauer beeinflussen. Da Patientendaten in grösserem Umfang weitergegeben werden, ist es schwierig, eine Akte nach 20 Jahren vollständig zu vernichten. 
contourner l'écueil du délai relatif de trois ans évoqué plus haut - c'est le seul délai de dix ans qui s'applique.

\section{Quid des délais cantonaux?}

Il arrive que le droit cantonal prescrive des délais de conservation minimale, voire maximale. Par exemple, à Genève [5], la Loi sur la santé prévoit à son article 57: «Les éléments du dossier doivent être conservés aussi longtemps qu'ils présentent un intérêt pour la santé du patient, mais au moins pendant dix ans dès la dernière consultation. Si aucun intérêt prépondérant pour la santé du patient ou pour la santé publique ne s'y oppose, le dossier est détruit après vingt ans au plus tard.» La plupart des cantons n'ont pas (encore) adapté leur délai à la révision du CO. Toutefois, les délais éventuellement plus courts du droit cantonal ne peuvent pas - en tout cas à eux seuls - être invoqués par le médecin pour échapper à une éventuelle responsabilité découlant du droit fédéral. En d'autres termes, le médecin aura du mal à se défendre en avançant qu'il a détruit les preuves en sa faveur conformément au droit de son canton. Certes, le fardeau de la preuve repose sur le patient-demandeur s'agissant de la violation des règles de l'art médical, mais les experts appelés à trancher se fondent avant tout sur le dossier médical [6].

\section{Et le droit à l'oubli?}

Ces dernières années, le droit à l'oubli a connu un fort écho médiatique. Le citoyen a réalisé qu'il peut avoir intérêt à ce que des données le concernant soient supprimées lorsqu'elles sont obsolètes ou peuvent lui nuire. En Suisse, la Loi fédérale sur la protection des données, applicable également aux dossiers médicaux des cabinets privés, dicte la destruction des données personnelles lorsque leur conservation cesse d'être nécessaire à la réalisation du but initial ou lorsqu'elle se révèle disproportionnée au regard des intérêts en présence. Dans le contexte médical, ce principe n'a guère de portée; on peut supposer, à moins de déclarations contraires de sa part, que le patient a toujours intérêt à ce que des données sur sa santé lui soient accessibles via son médecin. De même, tant que l'action pénale et civile ne sont pas prescrites, le soignant a toujours intérêt à pouvoir se défendre utilement en invoquant les informations du dossier médical [7].

Professeure associée UNIL HEC

Professeure titulaire UNIGE Droit Bureau MAIL 4081

Faculté de droit

Boulevard du Pont-d'Arve 40 CH-1211 Genève 4

valerie.junod[at]unil.ch

\section{Que changera le dossier électronique?}

Le dossier électronique du patient (DEP) peut aussi influer indirectement sur la durée de conservation. D’une part, la législation prévoit une destruction des pièces du DEP au bout de vingt ans, sans que le début de ce délai ne soit vraiment clair. D’autre part, comme les données du DEP seront partagées plus largement, il peut être délicat pour un professionnel de la santé de détruire définitivement son dossier après vingt ans, sachant que le patient ou d'autres personnes en garderont des copies séparées. En effet, le premier ignore si le patient ou d'autres conservent l'entier du dossier. Or, ceci peut prétériter le médecin confronté à une action en responsabilité puisque les parties au procès n'auront pas forcément accès aux mêmes documents [8]. Bien sûr, ce risque existe aussi aujourd'hui, puisqu'un patient peut avoir obtenu une copie de son dossier "physique». Toutefois, avec le DEP, la volonté des patients de garder durablement leurs propres copies sera encouragée par la facilité de la démarche.

Que faut-il retenir? Les médecins qui gardent des dossiers en format papier seront vraisemblablement forcés à passer au format électronique. Garder la trace physique de chaque interaction avec un patient pendant vingt ans est laborieux. Le faire via des logiciels permet un processus plus fluide. On peut espérer que cette transition servira aussi les objectifs d'efficacité, de coordination et d'économicité attribués au DEP. L'évolution des pratiques médicales, informatiques et juridiques suggère toutefois que la situation actuelle ne constituera certainement pas le point final au délicat enjeu de conservation. Une note positive toutefois: les actions en responsabilité contre les professionnels de la santé demeurent rares en Suisse et lorsqu'elles surviennent, elles se déclenchent en principe rapidement après l'intervention contentieuse. Les cas où le médecin se trouve démuni parce que le dossier a entretemps été détruit devraient rester exceptionnels.

\section{Références}

1 Sur ce qui doit ou devrait figurer dans le dossier médical, voir Landolt H. Medizinische Dokumentationspflicht - quo vadis? HAVE 2016, pp. 9-19.

2 Pally Hofmann U. Nouveau droit de la prescription. Bull Med Suisses. 2018;99(5152):1825-1826.

3 Par exemple, le patient qui agit contre son médecin parce que ce dernier l'a mal informé des coûts restant à sa charge doit toujours respecter le délai de 10 ans.

4 Pour des précisions, voir R. Christinat, pp. 289-99.

5 Pour les réglementations des autres cantons romands, voir leurs législations sur la santé récapitulées par Lovis/Geissbuhler, Dossier médical informatisé. Revue médicale suisse. 2002(2):876.

6 Sur ces aspects délicats et controversés, voir Christinat $\mathrm{R}$, Le procès en responsabilité civile médicale. Helbing 2019, pp. 358-66; Jetzer L, Die ärztliche Dokumentationspflicht und der Beweis des Behandlungsfehlers. ZBJV 148/2012, pp. 309-38; Schmid M, Dokumentationspflichten der Medizinalpersonen Unfang und Folgen ihrer Verletzung. HAVE 2009. pp. 350-61.
7 Sur ce sujet, voir Erard F/Amey L. La destruction du dossier médical sur requête du patient sous l'angle du droit public, Réflexions romandes e droit de la santé, 2016, pp. 277-91.

8 Par exemple, le patient se plaint d'un traitement médical inadéquat sur la base d'une pièce (ex. analyse de laboratoire vieille de 21 ans) dont lui, le patient, a accès, mais que le médecin ne peut en revanche plus resituer dans son contexte plus large. 